Research Article

\title{
Outdoor-to-Indoor Channel Measurement and Coverage Analysis for 5G Typical Spectrums
}

\author{
Zhimeng Zhong (D), Jianyao Zhao, and Chao Li \\ Shanghai Huawei Technologies Ltd., Shanghai 201206, China \\ Correspondence should be addressed to Zhimeng Zhong; zhongzhimeng@huawei.com
}

Received 7 March 2019; Revised 28 May 2019; Accepted 30 July 2019; Published 16 September 2019

Guest Editor: Danping He

Copyright (c) 2019 Zhimeng Zhong et al. This is an open access article distributed under the Creative Commons Attribution License, which permits unrestricted use, distribution, and reproduction in any medium, provided the original work is properly cited.

\begin{abstract}
The fifth-generation (5G) mobile communications system will adopt the millimeter wave (mmWave) band for outdoor-to-indoor (O2I) coverage to achieve ultrahigh data rate. However, it is a challenging task because of the large path loss and almost total blocking by building walls. In this work, we performed extensive measurements on the O2I propagation at $3.5,4.9$, and $28 \mathrm{GHz}$ simultaneously by using a multiband channel sounder. We captured the path loss distribution and angular power arrival profiles. We also measured the penetration loss at $28 \mathrm{GHz}$ through different kinds of glass windows. The widely adopted ordinary glass windows introduce the penetration loss of 3 to $12 \mathrm{~dB}$ that is acceptable and makes mmWave O2I coverage feasible. But the lowemissivity (low-E) windows that will be more popular in the future introduce $10 \mathrm{~dB}$ higher loss. The measurement results in this work can help analyse and anticipate the O2I coverage by mmWave, which is important for the design and deployment of the $5 \mathrm{G}$ network.
\end{abstract}

\section{Introduction}

In the fifth-generation (5G) mobile communication system, the outdoor-to-indoor (O2I) coverage in urban areas is an important scenario for the network deployment. The base stations on building rooftops or towers provide O2I coverage for nearby buildings. Meanwhile, the millimeter wave (mmWave) band is a key feature in the $5 \mathrm{G}$ system that is expected to provide very large bandwidth to support extremely high data rates for the eMBB (enhanced mobile broadband) applications. The performance of coverage depends critically on the propagation characteristics of the radio channels, such as the large-scale fading and angular power spectra in the mmWave band. Therefore, the radio channel models in different frequency bands, especially the sub-6G (frequency band below $6 \mathrm{GHz}$ ) and mmWave bands, are critical for the design and deployment of the $5 \mathrm{G}$ base stations. This work can reveal the signal coverage of both the low- and high-frequency bands and also accurately compare the channel characteristics among the different frequency bands. Therefore, this work can be used to guide the deployment of the $5 \mathrm{G}$ network using different frequency band resources.
Whether the 5G mmWave band can satisfy the O2I communication link requirement and provide reliable coverage remains an open question. To address this issue, the field channel measurements on the signal strength path loss are critical for the analysis and evaluate the feasibility of O2I coverage in mmWave band. Furthermore, the measurement results can also provide useful guidance for the design and deployment of the $5 \mathrm{G}$ networks.

In this article, we firstly survey the current efforts in both industrial and academic communities on the channel characterization in candidate $5 \mathrm{G}$ spectra. The channel models are the enabling tools for the evaluation and deployment of 5G networks in realistic environments.

Then, in order to study the feasible approach for coverage in the future $5 \mathrm{G}$ networks, we present our measurement campaign and analysis on the large-scale fading of O2I downlink channels in both sub-6 $\mathrm{GHz}$ and $\mathrm{mmWave}$ bands $(3.5,4.9$, and $28 \mathrm{GHz})$. The propagation from an outdoor rooftop base station (BS) to positions in a room and several corridors with various depths into a building were measured using a multiband channel sounder. The path losses at the three frequency points are compared and evaluated for the 
O2I coverage. In addition, the horn antenna for $28 \mathrm{GHz}$ channel sounding was rotated at multiple positions and heights in a room to capture the angle of arrival of propagation paths. Thus, we can analyse and enhance coverage based on the spatial (angular) propagation characteristics of mmWave in the O2I channels.

The glass window penetration propagation loss is critical for designing and optimizing O2I coverage, which depends on the composition, thickness, and layers of windows and varies greatly over frequency. We measured the penetration loss at $28 \mathrm{GHz}$ through dozens of windows. The coverage in urban buildings is analysed according to our measurement results and the practical exterior windows in North America and Europe.

Finally, the O2I coverage using mmWave is discussed based on our channel measurement results and window market analysis. It is found that $28 \mathrm{GHz}$ signals can provide coverage in outer-ring rooms by propagation through exterior windows. However, mmWave signals cannot provide coverage for inner rooms and corridors due to severe blocking by concrete walls. In this case, the sub- $6 \mathrm{GHz}$ band such as $3.5 \mathrm{GHz}$ can provide good coverage.

\section{Related Work}

2.1. Current O2I Channel Measurement. The authors measured the propagation property in 3.5 and $6 \mathrm{GHz}$ frequencies according to the channel impulse response (CIR) of O2I links [1-3]. In [1], the authors performed the O2I channel measurements with $56 \times 32$ antenna elements at $3.5 \mathrm{GHz}$ with $100 \mathrm{MHz}$ bandwidth at different locations and routes. It was found that the three-dimensional (3D) multiple-inputmultiple-output (MIMO) channel which fully utilized the elevation domain could improve capacity and also enhance the contributing eigenvalue number in the O2I scenario. The authors in [2] presented the cluster-parameter-based analysis of an O2I MIMO measurement campaign which was carried out at $3.5 \mathrm{GHz}$ in an office. The authors observed that the multipath components (MPCs) within one cluster in MIMO channels could be divided into several clusters and each had MPCs with smaller power weighted multipath component distance. In order to catch the propagation characteristics of channels in O2I scenarios, the authors in [3] presented the results of the 3D MIMO measurement in urban microcellular (UMi) O2I scenario at $6 \mathrm{GHz}$ with the bandwidth of $100 \mathrm{MHz}$.

Similarly, the authors in $[4,5]$ also presented a low-cost channel sounder and post-processing algorithms suitable for investigating the 3D MIMO channels to obtain the elevation and azimuth characteristics from 2.52 to $2.54 \mathrm{GHz}$. The channel measurement provided the elevation and azimuth angular spreads under different heights in urban macrocellular (UMa) and UMi environments.

For the higher frequency bands of O2I scenarios, studies are found to cover many frequency bands, such as 10,28, and $60 \mathrm{GHz}$ [6-8]. Reference [6] studied the indoor coverage at different frequencies $(10,30$, and $60 \mathrm{GHz})$ in a single building with an outdoor-deployed base station (BS). The work illustrated the general trends of how coverage varied across the frequency range. But the $\mathrm{O} 2 \mathrm{I}$ coverage at $60 \mathrm{GHz}$ may be quite difficult for some building types and the throughput was seriously affected, depending on the wall materials, interior layout, and building size. In [7], the authors presented results for path loss, penetration loss for two different type of housing, including single-family unit and multistory brick building.

\subsection{Millimeter-Wave Channel Measurement and Modelling.} The industry has much interests in the higher frequency band and proposed a series of 3GPP technical reports, which contain the channel models for frequencies from 0.5 up to $100 \mathrm{GHz}$ [9-12]. Meanwhile, the additional considerations on building penetration loss modelling for the $5 \mathrm{G}$ system is also investigated in [13].

While in academic community, many researchers have also explored the property of the high-frequency channels. In [14], the authors presented the radio propagation measurements and analysis for the mmWave transmission for inbuilding and urban cellular communications in the $28 \mathrm{GHz}$ band. Based on the measurements, the spatiotemporal channel characteristics, such as multipath delay, angular statistics, and path loss were analysed and modelled. In [15], the authors considered the path loss modelling in UMi environments. The authors established a spatially consistent stochastic street-by-street path loss model which could correctly describe the path LOS as a function of the street orientation as well as the large variance observed for all the path loss model parameters. The authors in [16] investigated the intracluster channel characteristics of NLOS $28 \mathrm{GHz}$ channels in street canyon scenarios. The channel characteristics include cluster numbers, number of subpaths within each cluster, intracluster delay spreads, and intracluster angular spreads.

The $38 \mathrm{GHz}$ mmWave frequency band is also a strong candidate for the future deployment of the $5 \mathrm{G}$ system. In [17], the authors presented a detailed measurement-based analysis of urban outdoor and O2I propagation characteristics at $38 \mathrm{GHz}$. The measurement results confirmed that, at this particular frequency, propagation in urban scenarios was mainly driven by LOS and reflection.

The above works have performed extensive channel measurements in both the lower-frequency bands and the mmWave band in different scenarios. However, the O2I coverage by using the mmWave band has been less explored. In this paper, we measured the path loss as well as the spatial power arrival profiles by using a triple-frequency channel sounder. This multiband channel sounding scheme enables us to compare and accurately analyse the propagation characteristics in different bands. We have also investigated the glazing markets in several countries and measured the penetration loss through a dozen of different types of windows. The results are critical for the design and deployment of the $5 \mathrm{G}$ system to achieve O2I coverage.

\section{Multiband O2I Measurement and Comparison}

3.1. Multiband Channel Sounder. The transmitter (TX) is comprises three radio frequency $(\mathrm{RF})$ chains at 3.5, 4.9, and 
$28 \mathrm{GHz}$, as show in Figure 1(a). Every RF chain consists of a signal generator, power amplifier, and transmitting antenna. Based on the narrow-band sounding scheme, each chain generates and transmits a single tone at the target frequency. The two white cylindrical antennas on the right are for 4.9 and $3.5 \mathrm{GHz}$ with the gain of 0.5 and $4 \mathrm{dBi}$, respectively. They are omnidirectional in the azimuth plane and have a halfpower beam width (HPBW) of $40^{\circ}$ in the elevation plane. The horn antenna on the left works at $28 \mathrm{GHz}$ with a gain of $8.5 \mathrm{dBi}$. It has $110^{\circ}$ and $40^{\circ} \mathrm{HPBW}$ in the azimuth and elevation plane, respectively. The parameters of TX antennas are summarized in Table 1.

The receiver $(\mathrm{RX})$ is equipped with two types of antennas to capture the sounding signal, as shown in Figure 1(b). Firstly, a wideband antenna can receive signals at 3.5, 4.9, and $28 \mathrm{GHz}$ with the gain of $4,0.5$, and $2.5 \mathrm{dBi}$, respectively. It is omnidirectional in the azimuth plane and has a HPBW of $40^{\circ}$ in the elevation dimension. Secondly, we use a horn antenna to capture the probing signals at $28 \mathrm{GHz}$ that has a $25 \mathrm{dBi}$ gain and $10^{\circ} \mathrm{HPBW}$ in both the azimuth and elevation planes. It is mounted on a numerical-control turntable to rotate around and capture the impinging signal from different directions. Also to better express it, the parameters of RX antennas are summarized in Table 1. In the field measurements on propagation path loss, the three RF chains in the TX transmit signals simultaneously and the multiband sounding signal received on the wideband antenna is input into a portable spectrum analyzer (SA). The SA has been programmed to record the received power at the three marked frequency points for 50 times continuously with the interval of 300 millisecond (ms). Then, it calculates the average power at each frequency and transfers the data to a computer for storage.

As shown in Figure 1, the signal-transmitting/ signalreceiving systems at the three frequencies are placed together at the same positions in order to compare the propagation characteristics accurately, as for the horn antenna on the RX side, by adjusting the horizontal and vertical angel of the horn antenna, firstly to find the direction with largest receive power. Then, the vertical tilt angle is fixed, and the horn antenna is rotated in the horizontal plane by a circle for capturing power in different directions.

\subsection{Multiband O2I Propagation Measurement Scenario.} The measurement campaign was conducted in a typical urban microcell O2I scenario in Shanghai, China. The TX was installed on the top of five-story building to emulate a BS. The height of the antennas was about 26 meters. The RX, emulating a UE, was moved on the 5th and 7 th floors in a modern apartment building on the opposite side of the TX, as shown in Figure 2. The heights of the two stories were 17 and 23 meters. The line-of-sight (LOS) direction from the $\mathrm{TX}$ to the room for measurement was about $10^{\circ}$ to the east, and the distance from the TX to the window at the 7 th floor was only 20 meters. On either floor, the RX was moved at 8 positions in a room and 40 positions in four corridors, as annotated in the floorplan in Figure 3. To analyse the propagation characteristics, the measurement routes were divided into four regions. The first region was the room where the RX was placed at 8 positions ( $\mathrm{P} 1$ to $\mathrm{P} 8$ ) along a line with a spacing of 1 meter between adjacent positions. The second region was a horizontal corridor next to the outerring rooms including the measurement positions from P11 to P23. The third region included two vertical corridors which had the measurement positions from P24 to P29 and from P34 to P40 excluding P35 and P36. Finally, the fourth region was a horizontal corridor in the inner part of the building, which included P29 to P36. The spacing between adjacent positions in the corridors was 2 meters.

3.3. Measurement Results of Multiband O2I Coverage. The contour diagrams in Figure 3 demonstrate the distributions of the pass loss magnitudes at the three frequencies of 3.5, 4.9 , and $28 \mathrm{GHz}$. For Area 1 (an outer room), the signals can propagate through the window and achieve good coverage at all the three frequencies. According to the link budget design, the maximal tolerable path loss for the BS-to-UE links is $\mathrm{PL}_{\text {th }}=140 \mathrm{~dB}$. The maximal tolerable path loss of $140 \mathrm{~dB}$ is widely used in the industry $[18,19]$. Actually, it will be slightly different for different frequencies and different companies. Generally, this tolerable path loss is used for brief evaluation of link budget during the design of wireless communication system or architecture. We can see that the path loss in the room is much smaller than $\mathrm{PL}_{\text {th }}$ at 3.5 and $4.9 \mathrm{GHz}$. For $28 \mathrm{GHz}$, the path loss satisfies the requirement in almost all the room but is just close to $\mathrm{PL}_{\text {th }}$ near the door. Therefore, the BS can cover the room entirely at all the three frequencies. How the $28 \mathrm{GHz}$ signals propagate in space and cover the room will be discussed in detail later.

In Area 2 (the long corridor in the middle), the path loss at all the three frequencies is obviously larger than that in Area 1 and increases with the horizontal depth along the corridor. It is noticed that the path loss is the smallest at position RX 11 . This is because there is a high-rise building to the west, as shown in Figure 2. Thus, some signals are reflected into the corridor through the window near $\mathrm{RX} 11$, resulting in the decreasing trend of the path loss from the west to the east. In addition, near the door of each room, the path loss decreases slightly. This is because the signals from the BS arrive at these positions through a window and a door. Hence, the path loss near the doors is smaller than that at other positions where the signals need to pass through two walls.

The average path loss in Area 2 at 4.9 and $28 \mathrm{GHz}$ is larger by 12 and $30 \mathrm{~dB}$, respectively, than that at $3.5 \mathrm{GHz}$. As shown in Figure 3, the path loss at the two sub-6 GHz frequencies is well below $\mathrm{PL}_{\text {th }}$, ensuring good coverage in Area 2. But the $28 \mathrm{GHz}$ band cannot meet the path loss requirement except the small part near the west window. As mentioned above, this is due to the paths reflected by a building outside the window.

Area 3 is the two vertical corridors, where the path loss is larger than that in Area 2, as shown in Figure 3. The average path loss at 4.9 and $28 \mathrm{GHz}$ is larger by 12 and $40 \mathrm{~dB}$, respectively, than that at $3.5 \mathrm{GHz}$. In Figures 3 (a) and 3(b), we can see that the path loss in the corridor on the right hand side (to the east) is obviously smaller than that in the left corridor 


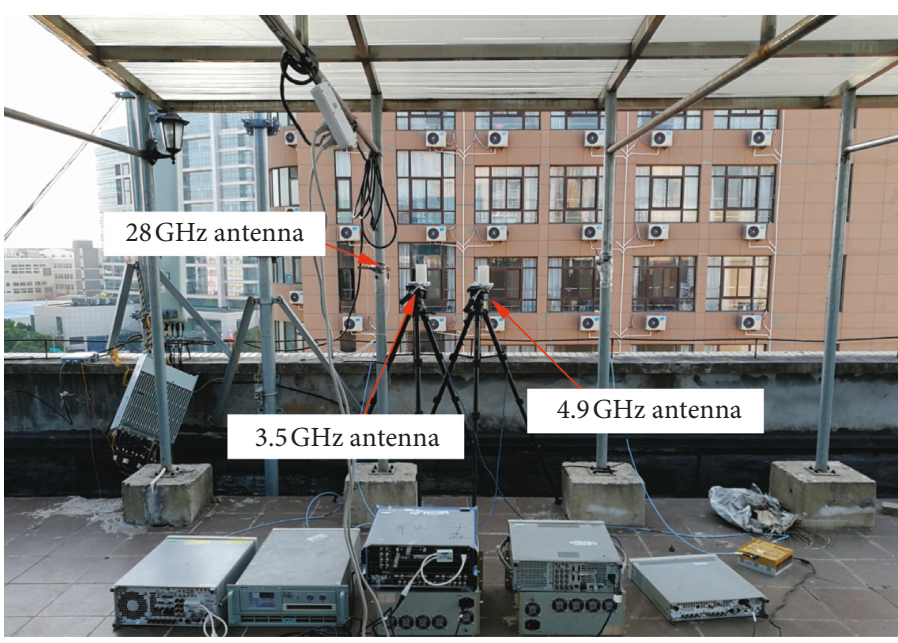

(a)

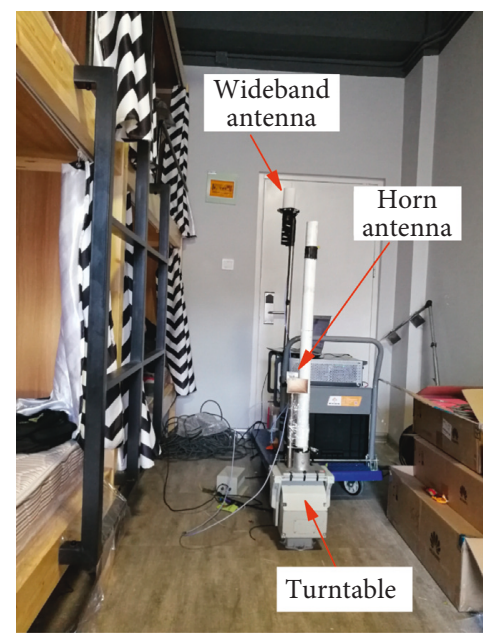

(b)

Figure 1: Multiband channel sounder at 3.5, 4.9, and $28 \mathrm{GHz}$. (a) Transmitter with three RF chains and antennas. (b) Receiver with two antennas.

TABLE 1: The configuration of antennas.

\begin{tabular}{lcccc}
\hline Class & Subclass & HPBW in horizontal plane $\left(^{\circ}\right)$ & HPBW in elevation plane $\left(^{\circ}\right)$ & Antenna gain $(\mathrm{dBi})$ \\
\hline \multirow{3}{*}{ TX antenna } & $3.5 \mathrm{GHz}$ TX antenna & 360 & 40 & 4 \\
& $4.9 \mathrm{GHz}$ TX antenna & 360 & 40 & 0.5 \\
& $28 \mathrm{GHz}$ TX antenna & 110 & 40 & 40 \\
RX antenna & $3.5 \mathrm{GHz}$ RX antenna & 360 & 40 & 4 \\
& $4.9 \mathrm{GHz}$ RX antenna & 360 & 40 & 0.5 \\
& $28 \mathrm{GHz}$ RX antenna type 1 & 360 & 10 & 2.5 \\
\hline
\end{tabular}

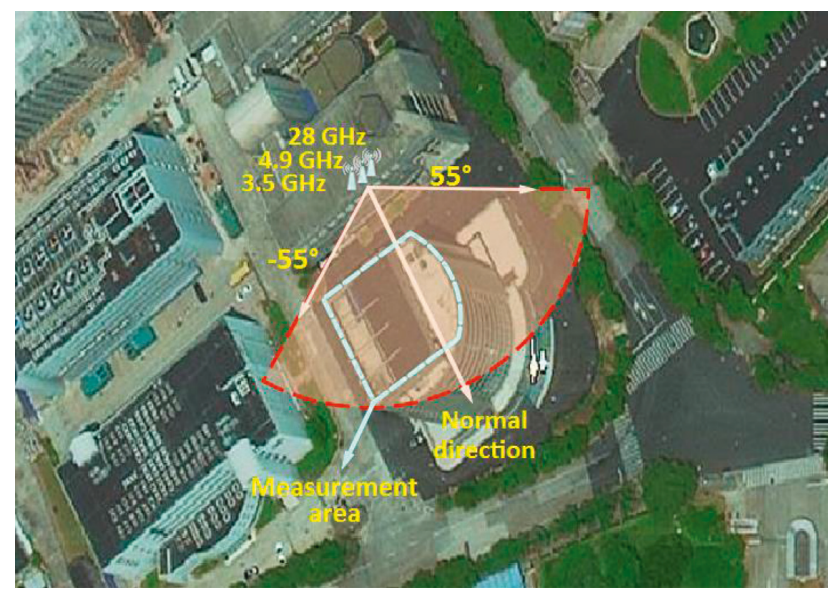

FIgURE 2: Multiband O2I channel measurement scenario.

(to the west). This is because, as mentioned earlier, the BS is to the east with respect to Area 1, and thus is closer to the right corridor. The signals at the sub- $6 \mathrm{GHz}$ frequencies can penetrate the walls and arrive at the two corridors. Since the propagation distance to the right corridor is shorter, the path loss is smaller. However, there is no obvious difference for the two corridors at $28 \mathrm{GHz}$ in Figure 3(c), as the mmWave signals cannot penetrate the walls. It is found that 3.5 and $4.9 \mathrm{GHz}$ can achieve full coverage over Area 3 , but $28 \mathrm{GHz}$ cannot satisfy the path loss requirement any more.
Area 4 is the horizontal corridor at the bottom. The distribution of the path loss in Area 4 is similar with that in Area 2. The path loss is relatively small near the window to the west end and increases along the corridor to the east. The average path loss at 4.9 and $28 \mathrm{GHz}$ is larger by 12 and $35 \mathrm{~dB}$, respectively, than that at $3.5 \mathrm{GHz}$. Since the path loss is still below $\mathrm{PL}_{\mathrm{th}}, 3.5$ and $4.9 \mathrm{GHz}$ can achieve full coverage for Area 4. But $28 \mathrm{GHz}$ can only cover the area near the window.

By comparison, it can be found that the difference between the pass loss at 3.5 and $4.9 \mathrm{GHz}$ does not change, which is always $12 \mathrm{~dB}$. This indicates that the penetration loss through the windows or walls is quite similar for sub$6 \mathrm{GHz}$ bands. However, the path loss difference between 3.5 and $28 \mathrm{GHz}$ varies in the areas. The difference becomes larger when the RX is moved to the inner part of the building. This indicates that the penetration loss of mmWave signals through walls is much larger.

In summary, we can see that the $28 \mathrm{GHz}$ band can provide coverage for indoor areas near exterior windows, such as outer rooms and ends of corridors. The propagation through windows results in the received power and hence determines the O2I coverage. Meanwhile the received power decays quickly with the depth of the indoor position due to large path loss. Consequently, mmWave cannot cover inner space of buildings without direct propagation through exterior windows. On the contrary, the sub- $6 \mathrm{GHz}$ bands can provide reliable coverage for the whole building in the UMi 


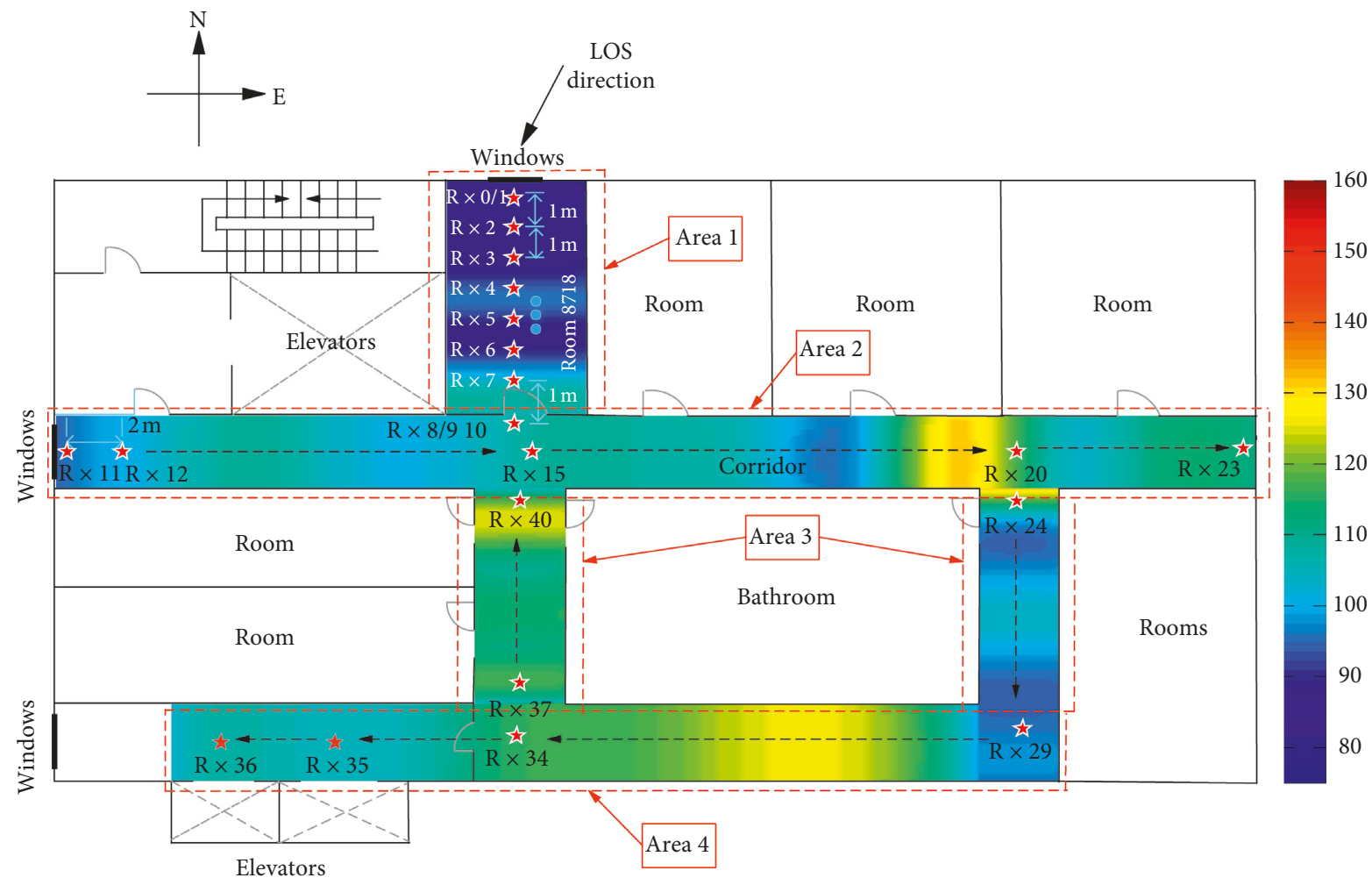

(a)

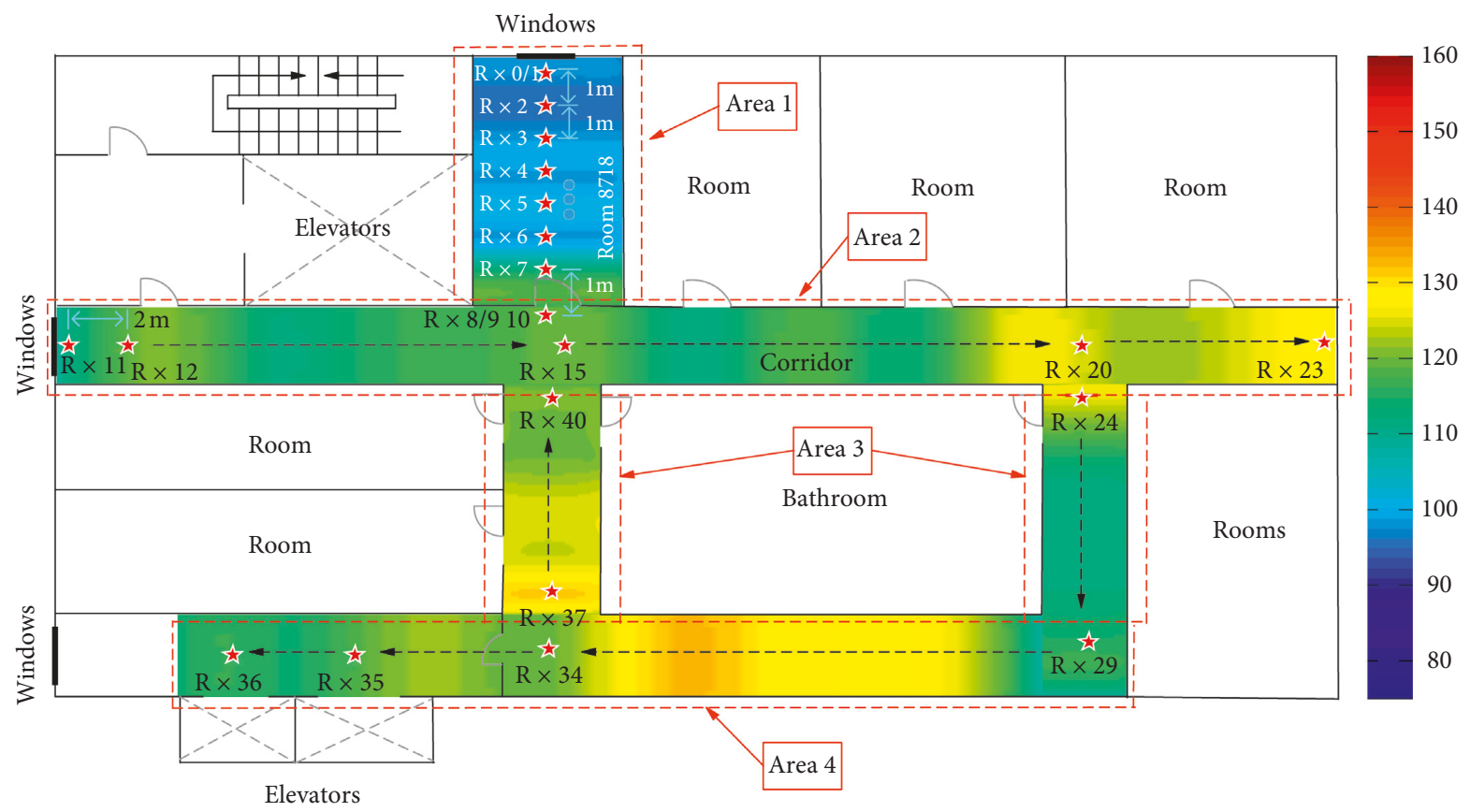

(b)

Figure 3: Continued. 


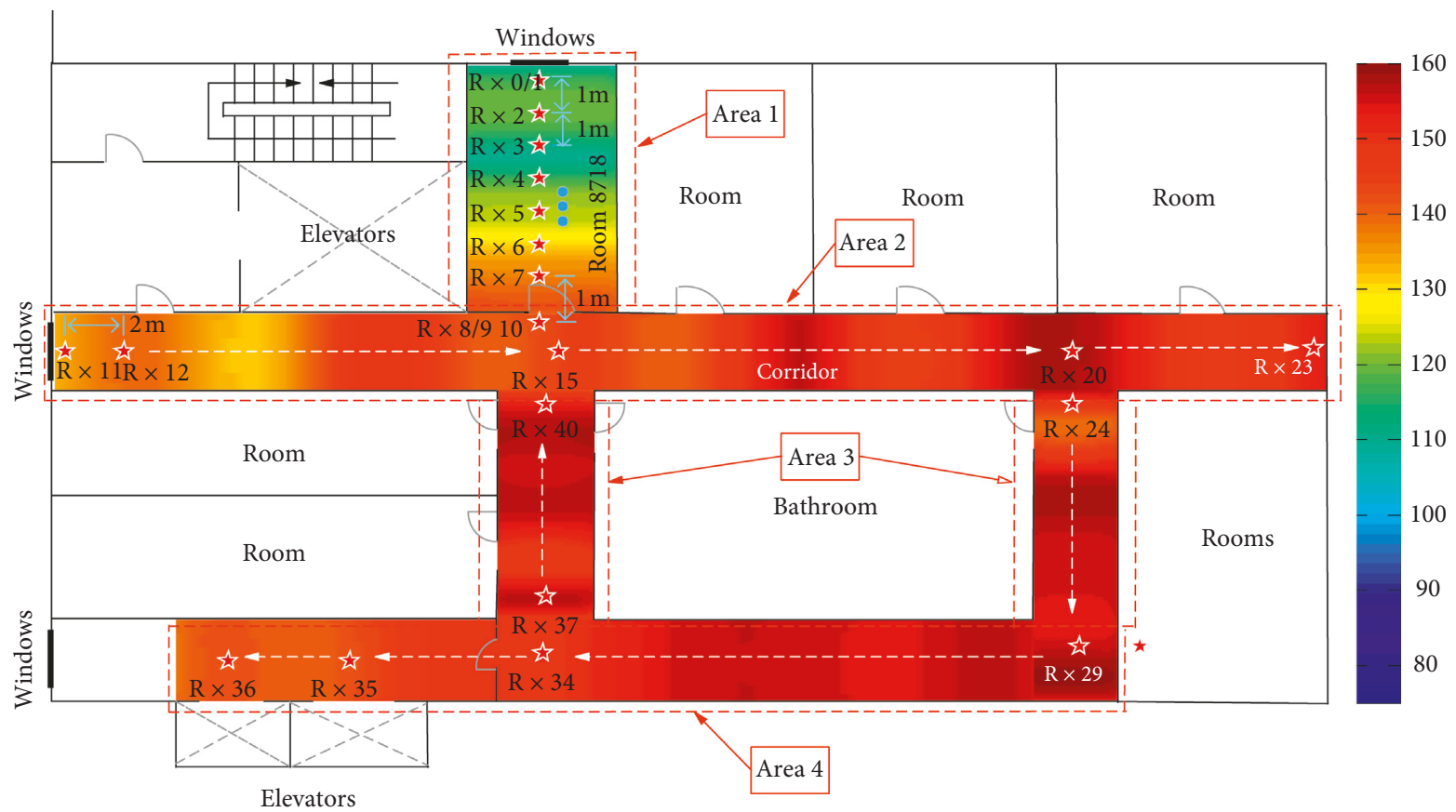

(c)

Figure 3: Measurement results for multiband O2I coverage. (a) Path loss on the 7th floor at $3.5 \mathrm{GHz}$. (b) Path loss on the 7th floor at $4.9 \mathrm{GHz}$. (c) Path loss on the 7th floor at $28 \mathrm{GHz}$.

O2I scenario, thanks to the good propagation characteristics of wall penetration and much smaller path loss.

\subsection{Spatial Propagation Measurement for O2I Coverage at} $28 \mathrm{GHz}$. As discussed in the previous subsection, the indoor coverage is achieved by the signal propagation through exterior windows. In this subsection, we study how the mmWave signals propagate in a room and are reflected inside to achieve indoor coverage. For this purpose, we performed the spatial propagation measurements on the $28 \mathrm{GHz}$ signals using the steering antenna method. As mentioned in Section 3.1, a $28 \mathrm{GHz}$ horn antenna was mounted on a turntable. The RX was placed 1, 2.5, and 5.5 meters away from the window along a straight line in the rooms on the 5 th and 7 th floors, as illustrated in Figure 3. At each position, the receiving antenna was raised to the heights of $0.5,1.5$, and $2.5 \mathrm{~m}$. It was rotated around with the step of the HPBW of $10^{\circ}$, to capture the received power at the directions [20]. As illustrative examples, the angular power profiles at the three measurement positions are plotted in Figure 4.

As can be seen in Figure 4(a), there are three significant clusters. The first one is at the angle of arrival (AoA) of $10^{\circ}$, which is the direction of the LOS path. Hence, this is the direct propagation cluster through the glass window. The second cluster is at the AoA of about $330^{\circ}$. It is the reflection from the wall on the left, which is coincident with the geometric relationship. Its power is slightly smaller than that of the LOS cluster due to the reflection loss. The third cluster is at the AoA of about $190^{\circ}$, which should be the reflection on the back wall of the room. Its power is smaller than the other two clusters because of longer propagation distance and reflection loss.
As shown in Figure 4(b), when the RX was moved to the second position (with more depth into the room), the power of the LOS cluster decays more than the reflection cluster by the left wall. This is because the LOS path is partially blocked but the wall reflection is not affected much and the power is still quite significant. Finally, at the third position, the power of the LOS cluster further reduces and the wall reflection cluster still has large power, as shown in Figure 4(c). Meanwhile, because the RX is now very close to the back wall of the room, the reflection by the metal door becomes quite significant. Also, we can see many scattered clusters in the power arrival profile from different directions, which should be generated by the reflections on the walls. In addition, it is found that, at the same $\mathrm{RX}$ position, the angular power arrival profiles at different heights are identical and do not change with height.

The results show that $28 \mathrm{GHz}$ band can provide full coverage over the rooms that are close to an outdoor BS. The LOS cluster contributes most power when the RX is close to the window, but it decays quickly when the RX moves away from the window. The one-bound reflection on a wall always generates a significant cluster, no matter the position of the $\mathrm{RX}$, which plays an important role in indoor coverage, even more than the LOS cluster. The mmWave signals through the window also experience intensive reflections inside the room and make a significant contribution in the received power, especially when the RX is located deep inside the room.

3.5. Path Loss Results of O2I Propagation at Different Frequencies. In order to predict the indoor coverage in the sub-6 GHz and mmWave bands, we evaluate the fitting curve of path loss in the O2I channels based on our 


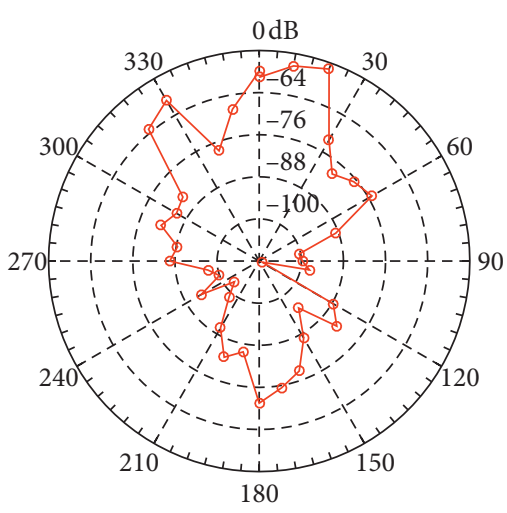

(a)

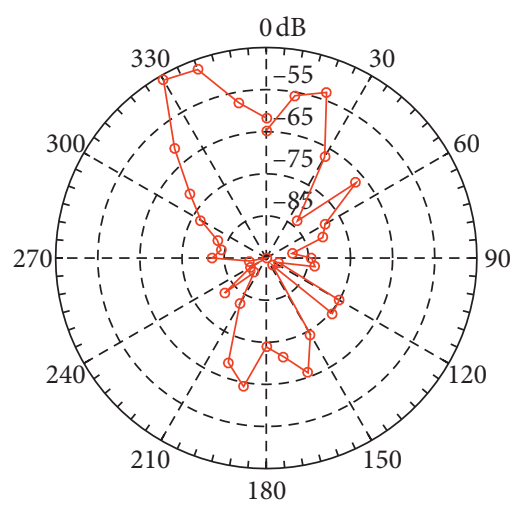

(b)

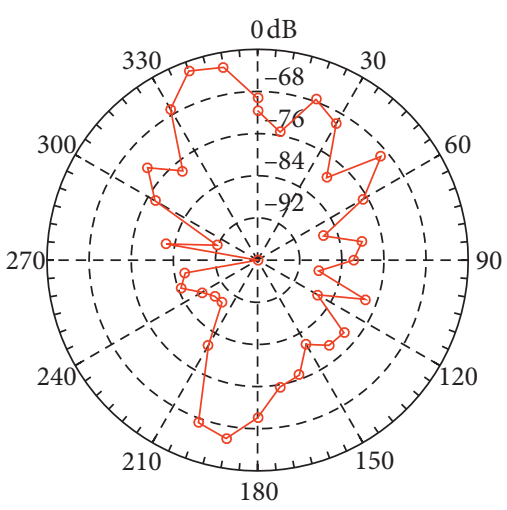

(c)

Figure 4: Measurement results of the angular power arrival profiles in the O2I channels at $28 \mathrm{GHz}$ ( “ $D$ ” is the distance between the RX and the window. " $H$ " is the height of the RX horn antenna.). (a) $D=1 \mathrm{~m}, H=1.5 \mathrm{~m}$; (b) $D=2.5 \mathrm{~m}, H=2 \mathrm{~m}$; (c) $D=5.5 \mathrm{~m}, H=0.5 \mathrm{~m}$.

measurement results. The path loss measurement results in Area 1, at the positions from RX 1 to RX 7 on the 7 th and 5 th floors, in Figure 3 are plotted in Figures 5(a) and 5(b), respectively, for easy analysis. The linear-function fitting model is also plotted. At first, the path loss increases basically linearly with the increase of the depth in the room. On the 7 th floor, as shown in Figure 5(a), the path loss exponents (slopes of the linear functions) are all about $1.5 \mathrm{~dB} /$ meter for the three frequencies. But the exponent becomes about $6 \mathrm{~dB} /$ meter for $28 \mathrm{GHz}$ at the 5 th floor.

This indicates that, when there is a clear LOS path, the path loss increments are identical for both sub-6 GHz and mmWave bands. The difference is mainly caused by the frequency gap. However, without a clear LOS path between the BS and UE (but there is a LOS path from the BS to the building window), the path loss for mmWave increases significantly. This may be because the signals cannot penetrate the wall and the received power is collected mainly by reflections. Thus, the path loss increases quickly when the RX moves deeply into the building.

Furthermore, the building penetration loss is another interesting parameter that required to be discussed. Here, we use results of RX 1 to RX 7 in Area 1 for such analysis since other $\mathrm{Rx}$ locations involve the deep indoor propagation scheme. For RX in Area 1, we obtain the building penetration loss by removing free space path loss from the whole receiver's path loss. It is shown that the averaging building penetration loss is $10.7 \mathrm{~dB}, 16.5 \mathrm{~dB}$, and $22.5 \mathrm{~dB}$ for $3.5,4.9$, and $28 \mathrm{GHz}$, respectively. As RX moves deeper into the room, the building penetration loss generally becomes larger. Since the window is not wide enough for all RX in Area 1 to have direct propagation path through glass window, the building penetration is due to hybrid of window and concrete walls.

\section{Glass Window Penetration Loss Measurement}

As revealed by the measurement results in Section 3, the coverage in the room at $28 \mathrm{GHz}$ depends mainly on the propagation through the exterior glass window. The signal power through the exterior walls is ignorable. Therefore, the propagation loss through glass windows is critical for $\mathrm{mmW}$ ave to realize O2I coverage. The loss is determined by the ingredients, thickness, and layers of glasses. Various types of glass windows are used in the global market. We have investigated the glazing type distributions in several countries, and the windows can be divided into three main categories: ordinary, low-E (low-emissivity), and IRR (infrared-reflective) glasses, based on the radiation energy differences and energy-saving requirements. Ordinary glass has good light transmission and can transfer almost all the indoor radiation and solar energy. Ordinary glass is mainly used in old-style residential and commercial areas with different thicknesses, colors, and layers. The light transmittance of low-E glass is slightly lower than that of ordinary glass, and the infrared light poorly penetrates. LowE glass can make room cold in summer and warm in winter with a good energy-saving effect. Low-E glass is mainly used in new residential and commercial areas, with a typical double and metal film and inert gas in the middle. IRR glass has poor transmittance for both visible and infrared light but a good energy-saving effect. IRR glass is mainly used for exterior walls of modern business office buildings.

Each category further includes a number of subtypes. To study the feasibility of mmWave O2I coverage for the $5 \mathrm{G}$ network, we selected nine typical kinds of glass windows to measure their penetration loss at $28 \mathrm{GHz}$. We utilized a vector network analyzer (VNA) with two horn antennas. The transmitting and receiving antennas were placed face to face on the two sides of a window. The penetration loss was obtained by comparing the received power measured with and without the window.

The measurement results and glazing distribution of the glass windows are listed in Table 2. It is found that the penetration loss varies greatly over the glass types. According to the measurement results in Section 3, the additional penetration loss by the window of $6 \mathrm{~dB}$ may reduce the indoor coverage depth only by 1 meter. The low-E glass windows in Shanghai and Vancouver both introduce about $15 \mathrm{~dB}$ loss, equivalently reducing the indoor coverage 


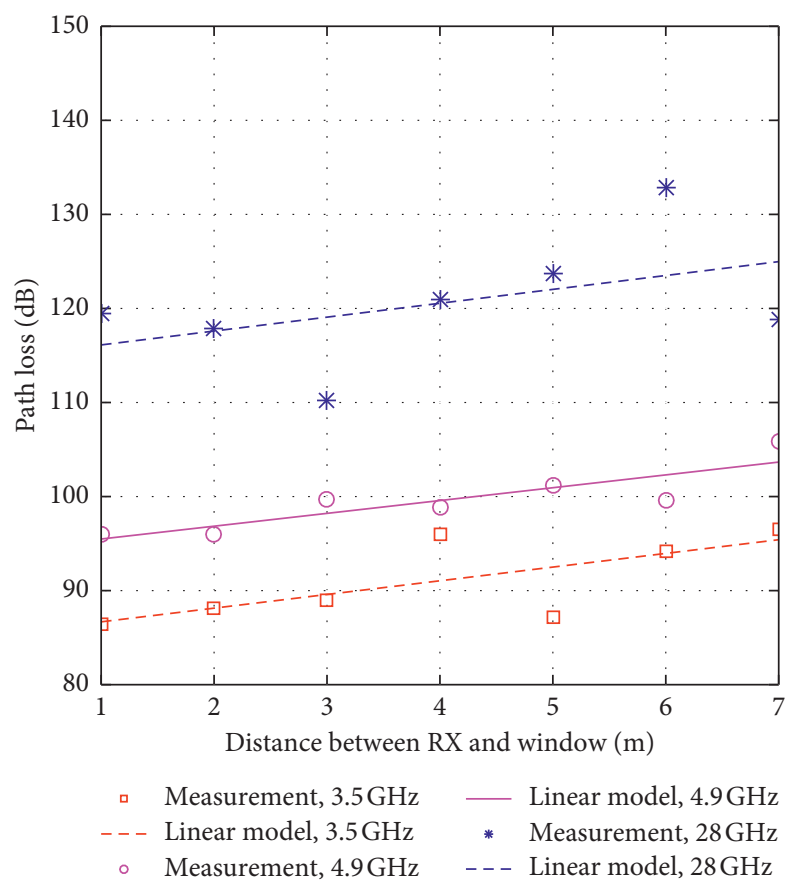

(a)

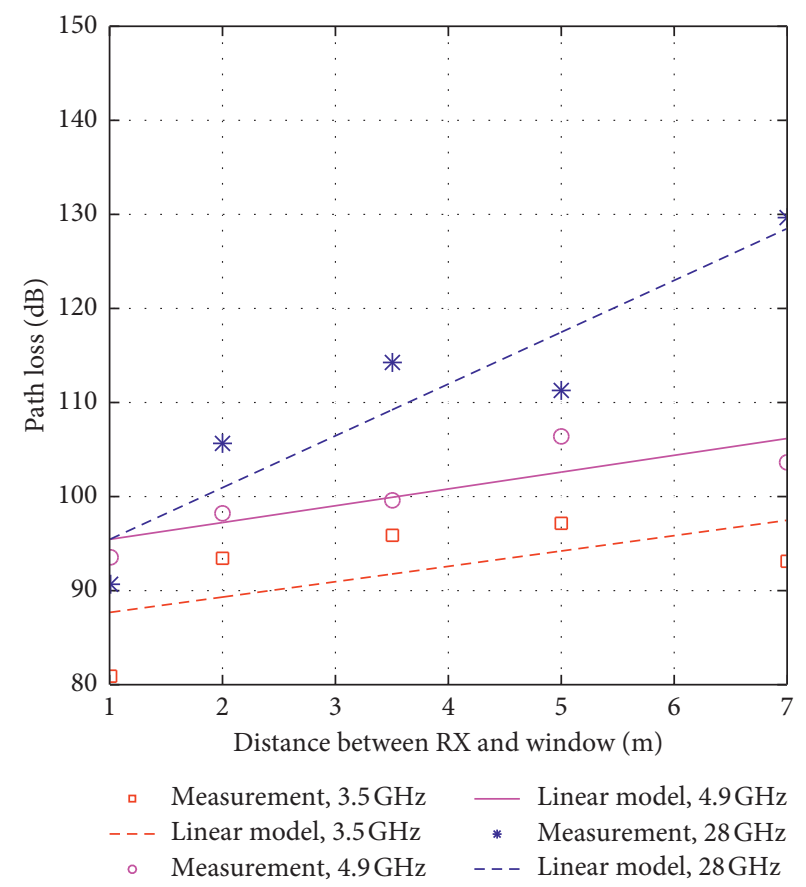

(b)

FIgURE 5: Measurement results of the path loss at different positions in the room at multiple frequencies. (a) The room on the 7th floor. (b) The room on the 5 th floor.

TABLE 2: Penetration loss of various types of glass windows.

\begin{tabular}{|c|c|c|c|c|}
\hline Class & Subclass & Penetration loss & Application scenario & Information [13] \\
\hline \multirow{4}{*}{ Ordinary glass } & Ordinary glass $(<15 \mathrm{~mm})$ & $3-8 \mathrm{~dB}$ & \multirow{4}{*}{$\begin{array}{l}\text { Old houses/shops (full coverage over rooms through } \\
\text { exterior windows) }\end{array}$} & \multirow{4}{*}{$\begin{array}{c}\text { Europe: } \sim 86 \% \\
\text { Austrian: }<20 \% \\
\text { Canada: } \sim 60 \% \\
\text { United States: } 80 \% \\
\text { China: } 45 \%-85 \%\end{array}$} \\
\hline & \multirow{3}{*}{ Coated glass $(6 \mathrm{~mm})$} & \multirow{3}{*}{$11.83 \mathrm{~dB}$} & & \\
\hline & & & & \\
\hline & & & & \\
\hline \multirow{4}{*}{ Low-E glass } & Shanghai, China (18 mm) & $15 \mathrm{~dB}$ & \multirow{4}{*}{$\begin{array}{c}\text { New/renovated homes/shops (coverage over areas } \\
\text { close to exterior windows) }\end{array}$} & Europe: $\sim 12 \%$ \\
\hline & & & & $\begin{array}{l}\text { Austrian: }>80 \% \\
\text { Canada: } \sim 30 \%\end{array}$ \\
\hline & Vancouver, Canada & $16 \mathrm{~dB}$ & & United States: $10 \%$ \\
\hline & & & & China: $10 \% \sim 50 \%$ \\
\hline \multirow{3}{*}{ IRR glass } & IRR ordinary window & $31.4 \mathrm{~dB}[13]$ & \multirow{3}{*}{ New CBD office building (O2I coverage is difficult) } & Europe: $\sim 2 \%$ \\
\hline & Modern IRR glass & $33 \mathrm{~dB}[13]$ & & Canada: $~ 10 \%$ \\
\hline & Tinted glass $(38 \mathrm{~mm})$ & $40.1 \mathrm{~dB}[21]$ & & $\begin{array}{c}\text { United States: } 10 \% \\
\text { China: } 5 \%\end{array}$ \\
\hline
\end{tabular}

depth by 2 to $3 \mathrm{~m}$. Therefore, the indoor coverage is still feasible for outer rooms. However, the IRR glass generates much larger loss from 31.4 to $40.1 \mathrm{~dB}$, and thus, the indoor coverage may be difficult. The ordinary glass is, obviously, most beneficial to O2I coverage by mmWave. As listed in Table 2, the ordinary glass is mostly widely used in the considered countries and occupies more than half of the markets [13]. Meanwhile, the IRR glass windows are adopted in less than $10 \%$ buildings.

As we can see, it is feasible to provide indoor coverage using mmWave in most areas at present, thanks to the low penetration loss and wide adoption of ordinary glass windows. However, low-E glass windows will be more and more adopted globally for better energy efficiency. For example, as shown in [13], the new or renovated residential buildings in Vancouver and Toronto all use low-E windows. The market occupancy of low-E window in China will increase by $15 \%$ to $20 \%$ per year and is anticipated to achieve 20\% in 2020 . Therefore, with the popularity of low-E windows, the O2I coverage by mmWave will become more challenging in the future.

In addition, it is also noticed that the uncoated glass generates loss of only 3 to $8 \mathrm{~dB}$, depending on its thickness (at most $15 \mathrm{~mm}$ ), glazing (single- or double-layered), and color. But the coated ordinary glass generates almost $12 \mathrm{~dB}$ loss even when it is only $6 \mathrm{~mm}$ thick. Hence, coating can 
significantly increase the penetration loss for ordinary glass windows.

\section{Conclusions}

The measurement results in this paper reveal the path loss distribution and coverage conditions at $3.5,4.9$, and $28 \mathrm{GHz}$ in a typical UMi O2I scenario. It can be seen that the signals at $28 \mathrm{GHz}$ propagate into the room and corridors mainly through the windows and doors. The path loss is smaller than the threshold of $140 \mathrm{~dB}$ and thus the outer rooms can be covered. But the path loss is too high to cover the inner part of the building $(>140 \mathrm{~dB})$. The average path loss at 4.9 and $28 \mathrm{GHz}$ is larger by 12 and $35 \mathrm{~dB}$, respectively, than that at $3.5 \mathrm{GHz}$. The sub-6G signals can penetrate the concrete wall much better and ensure good coverage for the whole building. The difference between the path loss at 3.5 and $4.9 \mathrm{GHz}$ is consistently $12 \mathrm{~dB}$. Based on the comparison, we can conclude that the mmWave band can meet the coverage requirement for outer rooms with exterior windows. The direct LOS cluster can provide required received power for the areas close to windows, but the reflection of mmWave signals by walls is more significant and ensures the coverage over the whole room. The penetration losses through uncoated glass windows of the ordinary, low-E, and IRR windows are 6,15 , and $35 \mathrm{~dB}$. The hybrid approach using both sub-6G and mmWave bands is promising to provide seamless coverage. To design the multiband access network and allocate the spectrum resource based on the propagation characteristics is an interesting and important research topic for $5 \mathrm{G}$.

\section{Data Availability}

The channel measurement data used to support the findings of this study are restricted in order to protect the commercial confidentiality. Data are available for researchers who meet the criteria for access to confidential data.

\section{Conflicts of Interest}

The authors declare that there are no conflicts of interest regarding the publication of this paper.

\section{References}

[1] J. Zhang, Y. Zhang, Y. Yu, R. Xu, Q. Zheng, and P. Zhang, “3D MIMO: how much does it meet our expectations observed from channel measurements," IEEE Journal on Selected Areas in Communications, vol. 35, no. 8, pp. 1887-1903, 2017.

[2] D. Du, J. Zhang, C. Pan, and C. Zhang, "Cluster characteristics of wideband 3D MIMO channels in outdoor-to-indoor scenario at $3.5 \mathrm{GHz}$," in Proceedings of the 2014 IEEE 79th Vehicular Technology Conference (VTC Spring), pp. 1-6, IEEE, Seoul, South Korea, May 2014.

[3] Q. Zheng, J. Zhang, H. Yu, Y. Zhang, and L. Tian, "Propagation statistic characteristic of 3D MIMO channel in outdoor-to-indoor scenario with different antenna heights," in Proceedings of the 2016 19th International Symposium On Wireless Personal Multimedia Communications (WPMC), pp. 411-416, IEEE, Shenzhen, China, November 2016.
[4] V. Kristem, S. Sangodoyin, C. U. Bas et al., "3D MIMO outdoor-to-indoor propagation channel measurement," IEEE Transactions on Wireless Communications, vol. 16, no. 7, pp. 4600-4613, 2017.

[5] R. Zhang, S. Wang, X. Lu, W. Duan, and L. Cai, "Two-dimensional DoA estimation for multipath propagation characterization using the array response of PN-sequences," IEEE Transactions on Wireless Communications, vol. 15, no. 1, pp. 341-356, 2016.

[6] E. Semaan, F. Harrysson, A. Furuskar, and H. Asplund, "Outdoor-to-indoor coverage in high frequency bands," in Proceedings of the 2014 IEEE Globecom Workshops (GC Wkshps), pp. 393-398, IEEE, Austin, TX, USA, December 2014.

[7] C. U. Bas, R. Wang, T. Choi et al., "Outdoor to indoor penetration loss at $28 \mathrm{GHz}$ for fixed wireless access," in Proceedings of the 2018 IEEE International Conference on Communications (ICC), vol. 99, pp. 1-6, IEEE, Kansas City, MO, USA, May 2018.

[8] L. Ahumada, E. Carreno, A. Angles, and D. Schkolnik, "Studying the dynamics of outdoor-indoor wireless links at $60 \mathrm{GHz}$," in Proceedings of the 2018 IEEE-APS Topical Conference on Antennas and Propagation in Wireless Communications (APWC), pp. 834-837, IEEE, Cartagena des Indias, Colombia, September 2018.

[9] ETSI, "Study on channel model for frequencies from 0.5 to $100 \mathrm{GHz}$ (release 14) (3GPP technical specification group radio access networks)," Tech. Rep. TR 38.901 V14.0.0, ETSI, Sophia Antipolis, France, 2017.

[10] ITU, Prediction of Building Entry Loss, Recommendation ITUR P.2109-0, ITU, Geneva, Switzerland, 2017.

[11] ITU, "Compilation of measurement data relating to building entry loss," Report ITU-R P.2346-2, ITU, Geneva, Switzerland, 2017.

[12] mmMAGIC, "Measurement results and final mmMAGIC channel models," H2020-ICT-671650-mmMAGIC/D2.2 v2.0, 2017, https://5g-ppp.eu/mmmagic/.

[13] Additional considerations on building penetration loss modeling for 5G system performance evaluation," Straight Path Communications, Tech. Rep. R1-163408, 2016.

[14] J. Ko, Y.-J. Cho, S. Hur et al., "Millimeter-wave channel measurements and analysis for statistical spatial channel model in in-building and urban environments at $28 \mathrm{GHz}$," IEEE Transactions on Wireless Communications, vol. 16, no. 9, pp. 5853-5868, 2017.

[15] A. Karttunen, A. F. Molisch, S. Hur, J. Park, and C. J. Zhang, "Spatially consistent street-by-street path loss model for $28 \mathrm{GHz}$ channels in micro cell urban environments," IEEE Transactions on Wireless Communications, vol. 16, no. 11, pp. 7538-7550, 2017.

[16] S. Wu, S. Hur, K. Whang, and M. Nekovee, "Intra-cluster characteristics of $28 \mathrm{GHz}$ wireless channel in urban micro street canyon," in Proceedings of the 2016 IEEE Global Communications Conference (GLOBECOM), pp. 1-6, IEEE, Washington, DC, USA, December 2016.

[17] I. Rodriguez, H. C. Nguyen, T. B. Sorensen et al., "Analysis of $38 \mathrm{GHz}$ mmwave propagation characteristics of urban scenarios," in Proceedings of European Wireless 2015; 21th European Wireless Conference, pp. 1-8, VDE, Budapest, Hungary, May 2015.

[18] J. S. Seybold, Introduction to RF Propagation, John Wiley \& Sons, Hoboken, NJ, USA, 2005. 
[19] J.-H. Park, K.-W. Cheun, W.-I. Roh, and J.-W. Cho, "Method and apparatus for transmitting or receiving rach signal in beamforming system," US20140348051A1, 2014.

[20] R. Zhang, Y. Zhou, X. Lu, C. Cao, and Q. Guo, "Antenna deembedding for mmWave propagation modeling and field measurement validation at $73 \mathrm{GHz}$ field measurement validation at $73 \mathrm{GHz}$," IEEE Transactions on Microwave Theory and Techniques, vol. 65, no. 10, pp. 3648-3659, 2017.

[21] H. Zhao, R. Mayzus, S. Sun et al., " $28 \mathrm{GHz}$ millimeter wave cellular communication measurements for reflection and penetration loss in and around buildings in New York city," in Proceedings of the 2013 IEEE International Conference on Communications (ICC), pp. 5163-5167, IEEE, Budapest, Hungary, May 2013. 


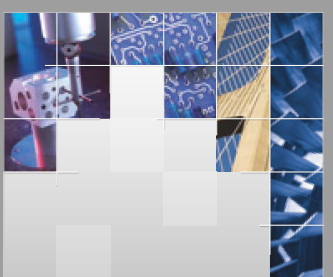

\section{Enfincering}
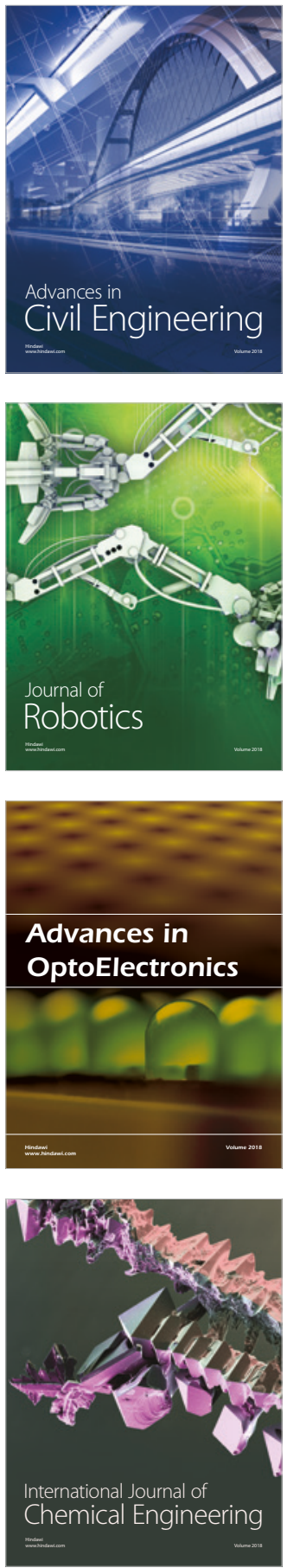

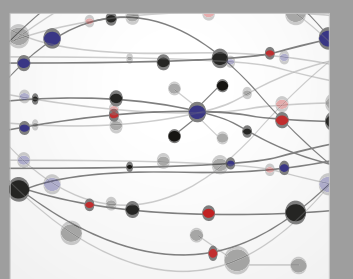

\section{Rotating \\ Machinery}

The Scientific World Journal

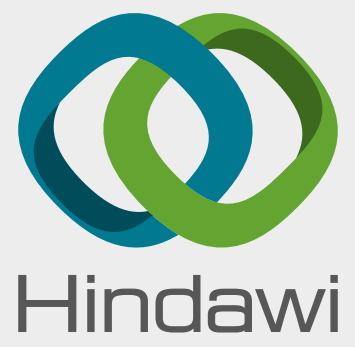

Submit your manuscripts at

www.hindawi.com
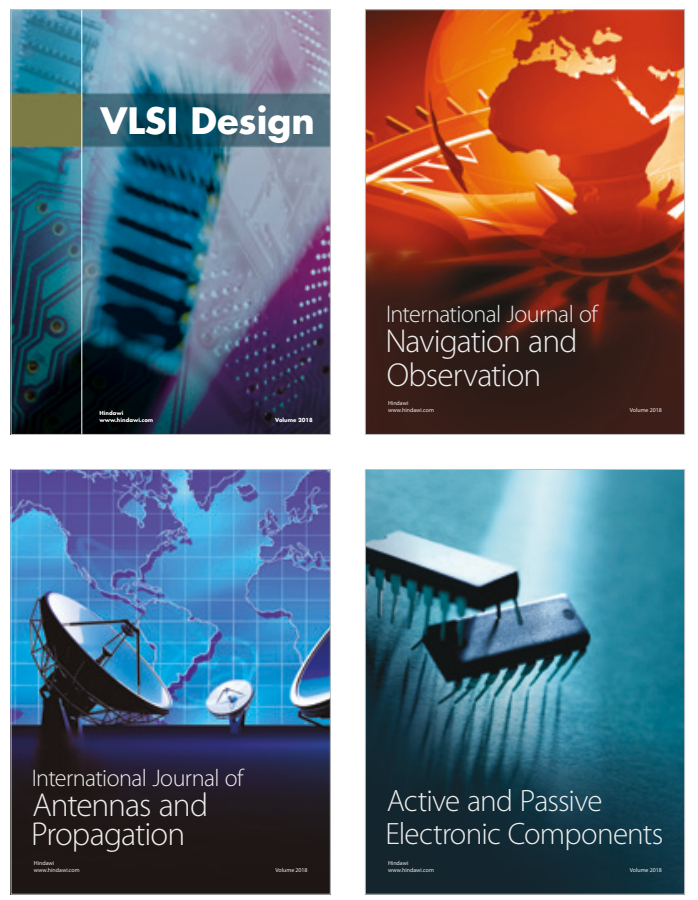
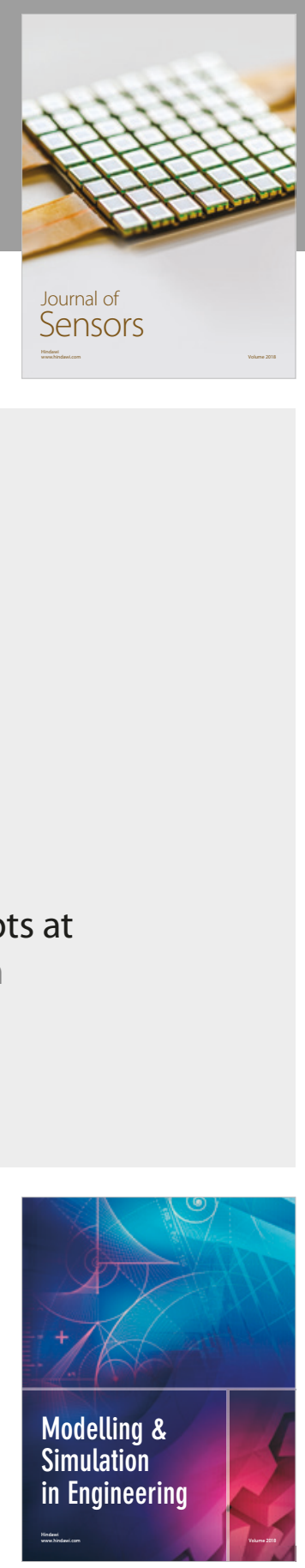

\section{Advances \\ Multimedia}
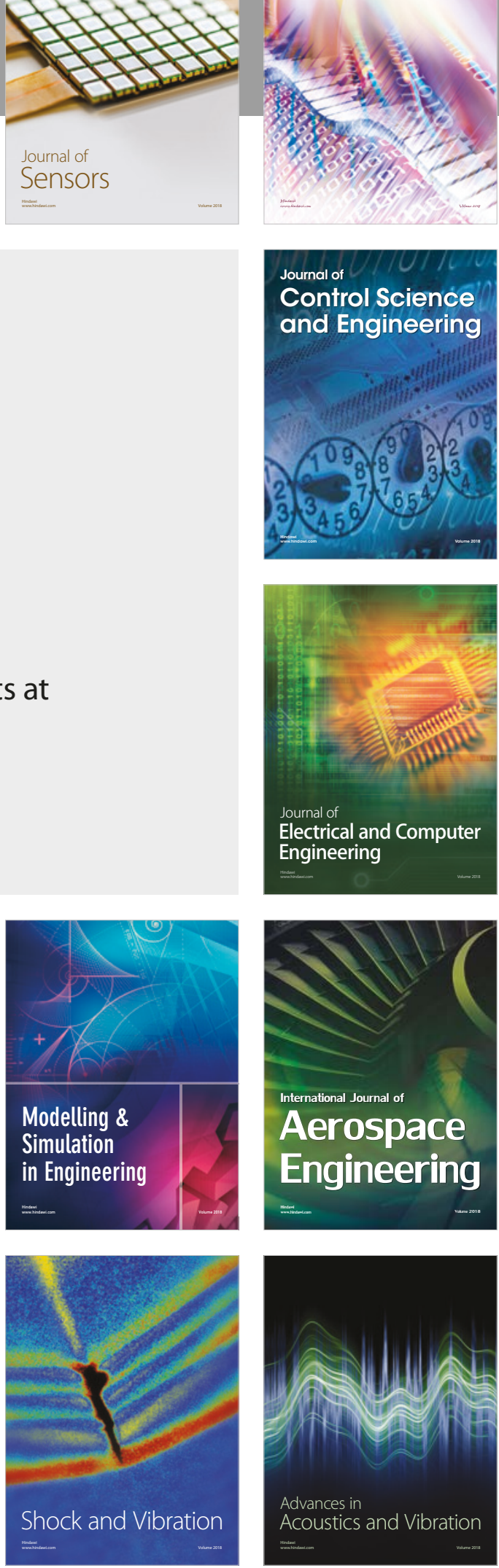